\title{
Nanolaminate-based design for UV laser mirror coatings
}

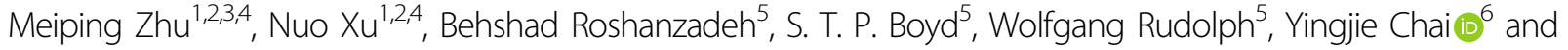 \\ Jianda Shao ${ }^{1,3,4}$
}

\begin{abstract}
With ever-increasing laser power, the requirements for ultraviolet (UV) coatings increase continuously. The fundamental challenge for UV laser-resistant mirror coatings is to simultaneously exhibit a high reflectivity with a large bandwidth and high laser resistance. These characteristics are traditionally achieved by the deposition of laser-resistant layers on highly reflective layers. We propose a "reflectivity and laser resistance in one" design by using tunable nanolaminate layers that serve as an effective layer with a high refractive index and a large optical bandgap. An $\mathrm{Al}_{2} \mathrm{O}_{3}-\mathrm{HfO}_{2}$ nanolaminate-based mirror coating for UV laser applications is experimentally demonstrated using ebeam deposition. The bandwidth, over which the reflectance is $>99.5 \%$, is more than twice that of a traditional mirror with a comparable overall thickness. The laser-induced damage threshold is increased by a factor of $\sim 1.3$ for $7.6 \mathrm{~ns}$ pulses at a wavelength of $355 \mathrm{~nm}$. This tunable, nanolaminate-based new design strategy paves the way toward a new generation of UV coatings for high-power laser applications.
\end{abstract}

The demand for laser-resistant mirror coatings is increasing in inertial confinement fusion (ICF) ${ }^{1}$, extreme light infrastructure ${ }^{2}$ and other laser applications ${ }^{3-6}$. An ideal ultraviolet (UV) laser mirror (UVLM) coating requires a high reflectivity with a large bandwidth and a high laser-induced damage threshold (LIDT). Unfortunately, these requirements are difficult to satisfy simultaneously, because, for example, a high reflectivity requires materials with a high refractive index $(n)$, while higher $n$ materials tend to have a smaller optical bandgap and therefore a lower LIDT. Traditionally, compromises are made for these seemingly contradictory requirements $^{7-10}$. We propose to use nanolaminate coatings for UVLMs. Nanolaminate materials ${ }^{11-16}$ have properties that make them attractive for many applications ${ }^{15,17-20}$. Our nanolaminate-based UVLM coatings are deposited

Correspondence: Meiping Zhu (bree@siom.ac.cn) or

Wolfgang Rudolph (wrudolph@unm.edu) or Jianda Shao (jdshao@siom.ac.cn)

'Laboratory of Thin Film Optics, Shanghai Institute of Optics and Fine Mechanics, Chinese Academy of Sciences, Shanghai 201800, China

${ }^{2}$ Center of Materials Science and Optoelectronics Engineering, University of Chinese Academy of Sciences, Beijing 100049, China

Full list of author information is available at the end of the article. using e-beam evaporation, a technique that is particularly favorable for large laser optics ${ }^{10,21-24}$. This novel concept results in improved performance parameters and paves the way toward a new generation of UV coatings for highpower laser applications.

In the traditional "reflectivity bottom and LIDT top" combination design (TCD coating) strategy, alternating high- $n$ and low- $n$ layers, such as $\mathrm{HfO}_{2}$ and $\mathrm{SiO}_{2}$, are deposited on the substrate to obtain a high reflectivity, as illustrated in Fig. 1a (the high- $n$ layer is denoted as $C_{H N}$ ). Subsequently, pairs of high- $n$ layers (with a relatively larger optical bandgap than $C_{H N}$, denoted as layer $C_{L B}$ ) and low- $n$ layers, such as $\mathrm{Al}_{2} \mathrm{O}_{3}$ and $\mathrm{SiO}_{2}$, or $\mathrm{LaF}_{3}$ and $\mathrm{AlF}_{3}$, are deposited to achieve a high $\mathrm{LIDT}^{8,9}$. Our new "reflectivity and laser resistance in one" strategy uses nanolaminate layers with co-evaporated interfaces, which are produced by alternating $\mathrm{C}_{L B}$ layers and $\mathrm{C}_{H N}$ layers, as shown in Fig. $1 \mathrm{~b}$. The $\mathrm{C}_{L B}-\mathrm{C}_{H N}$ nanolaminate layers can be considered high- $n$ layers, with a tunable refractive index and optical bandgap. As we will show, this arrangement allows for mirror designs with the advantageous combination of a high reflectivity and a high LIDT. 


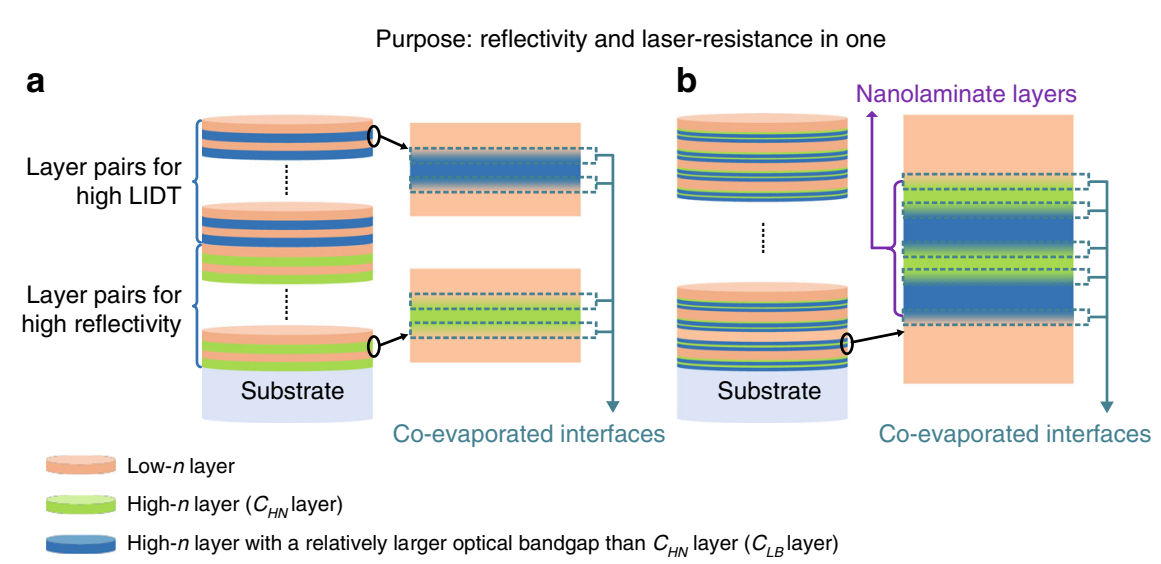

Fig. 1 Schematic diagram of the stacks of optical coatings for HR mirrors with a high LIDT. a Traditional design using pairs of high- and lowindex materials. $\mathbf{b}$ Proposed strategy using nanolaminate layers and low-index materials

The high- $n$ materials $\mathrm{HfO}_{2}$ and $\mathrm{Al}_{2} \mathrm{O}_{3}$ in combination with the low- $n$ material $\mathrm{SiO}_{2}$ are widely used for multilayer coating stacks in the UV region ${ }^{25}$. The microstructure and optical properties of an e-beam-evaporated $\mathrm{Al}_{2} \mathrm{O}_{3}-\mathrm{HfO}_{2}$ nanolaminate coating consisting of 18 $\mathrm{Al}_{2} \mathrm{O}_{3}-\mathrm{HfO}_{2}$ pairs have been compared with those of $\mathrm{HfO}_{2}$ and $\mathrm{Al}_{2} \mathrm{O}_{3}$ single-layer coatings. In this study, 4$\mathrm{nm}$ co-evaporated interfaces are introduced for each alternating interface between two different materials, as shown in Fig. 1. The elemental percentages of an $\mathrm{Al}_{2} \mathrm{O}_{3}-\mathrm{HfO}_{2}$ pair in the nanolaminate vs. depth are measured by XPS and are shown in Fig. 2a. Due to diffusion, both the $\mathrm{Al}_{2} \mathrm{O}_{3}$ and $\mathrm{HfO}_{2}$ contents are observed at each depth. The $\mathrm{Al}_{2} \mathrm{O}_{3}$ to $\mathrm{HfO}_{2}$ integrated content ratio in an $\mathrm{Al}_{2} \mathrm{O}_{3}-\mathrm{HfO}_{2}$ pair is calculated to be $\sim 1.7: 1$. A sharp diffraction peak is not observed in the X-ray diffraction (XRD) spectra of the $\mathrm{Al}_{2} \mathrm{O}_{3}-\mathrm{HfO}_{2}$ nanolaminate and $\mathrm{Al}_{2} \mathrm{O}_{3}$ single-layer coatings, while multiple sharp diffraction peaks indicative of crystallinity are obtained from the $\mathrm{HfO}_{2}$ single-layer coating (Fig. 2b). The transmittance spectra of the substrate and coatings are measured to determine the refractive indices (Fig. 2c). The optical bandgaps of the $\mathrm{Al}_{2} \mathrm{O}_{3}$ and $\mathrm{HfO}_{2}$ single-layer coatings and $\mathrm{Al}_{2} \mathrm{O}_{3}-\mathrm{HfO}_{2}$ nanolaminate coatings are estimated using the Tauc equation ${ }^{26}$ to be $6.25 \mathrm{eV}, 5.44 \mathrm{eV}$, and $5.65 \mathrm{eV}$, respectively. The $\mathrm{Al}_{2} \mathrm{O}_{3}-\mathrm{HfO}_{2}$ nanolaminate coating acts as an equivalent single-layer coating with a higher refractive index than $\mathrm{Al}_{2} \mathrm{O}_{3}$ and a larger optical bandgap than $\mathrm{HfO}_{2}$. The (average) refractive index and optical bandgap can be tuned by adjusting the thickness ratio of the two materials in the nanolaminate layers while keeping the total optical thickness constant. This allows one to develop UVLM coatings with a high reflectivity and a high LIDT.

Using the refractive index and optical bandgap tunability, a nanolaminate-based multilayer coating (NLD
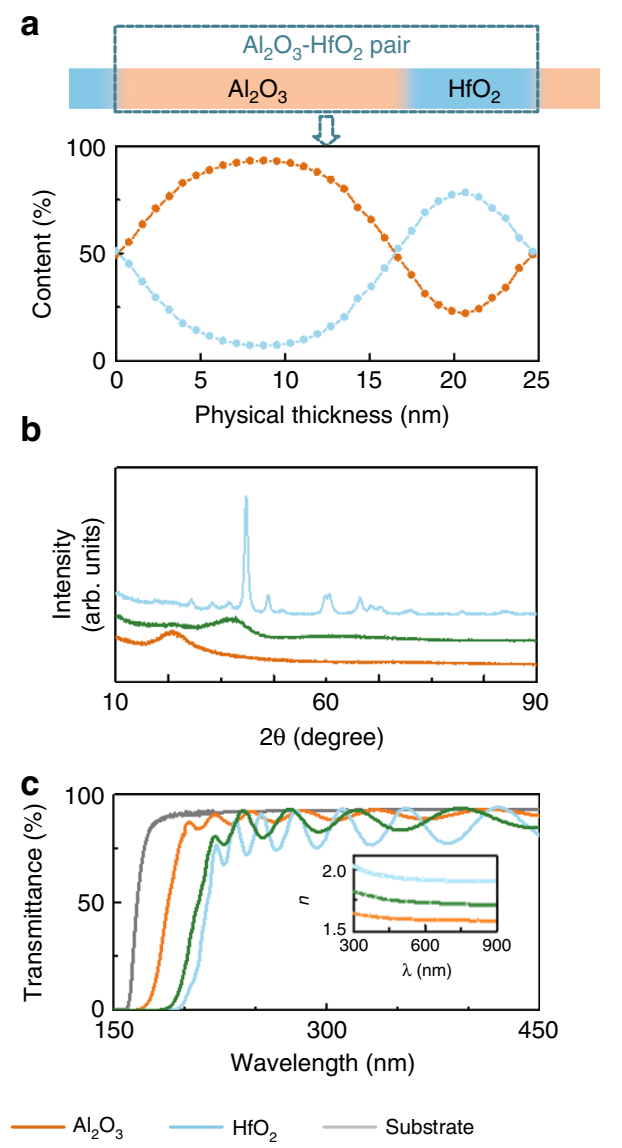

$-\mathrm{Al}_{2} \mathrm{O}_{3}-\mathrm{HfO}_{2}$ nanolaminate coating

Fig. 2 The microstructure and optical properties of $\mathrm{Al}_{2} \mathrm{O}_{3}-\mathrm{HfO}_{2}$ nanolaminate, $\mathrm{Al}_{2} \mathrm{O}_{3}$ and $\mathrm{HfO}_{2}$ single layer coatings. a $\mathrm{Al}_{2} \mathrm{O}_{3}$ and $\mathrm{HfO}_{2}$ content as a function of physical thickness in a nanolaminate coating. $\mathbf{b}$ XRD spectra and $\mathbf{c}$ transmittance of an $\mathrm{Al}_{2} \mathrm{O}_{3}$ single layer, an $\mathrm{HfO}_{2}$ single layer and an $\mathrm{Al}_{2} \mathrm{O}_{3}-\mathrm{HfO}_{2}$ nanolaminate coating with physical thicknesses of $513.8 \mathrm{~nm}, 540.7 \mathrm{~nm}$, and $444.1 \mathrm{~nm}$, respectively. The inset shows the corresponding refractive indices 
coating) consisting of $\mathrm{Al}_{2} \mathrm{O}_{3}-\mathrm{HfO}_{2}$ nanolaminate layers with an equivalent $n$ of 1.714 at $355 \mathrm{~nm}$ is designed. The coating structure is as follows: substrate $/ 4 \mathrm{~L}$ (0.335A0.165H0.335A0.165HL $)^{20} \mathrm{~A} 8.15 \mathrm{~L} /$ air. Here, A, H, and $\mathrm{L}$ represent $\mathrm{Al}_{2} \mathrm{O}_{3}, \mathrm{HfO}_{2}$, and $\mathrm{SiO}_{2}$ layers with a quarter-wavelength optical thickness (QWOT), respectively. The number represents the optical thickness in units of the QWOT of the respective material. Thus, $0.335 \mathrm{~A} 0.165 \mathrm{H} 0.335 \mathrm{~A} 0.165 \mathrm{H}$ represents a nanolaminate layer with four sublayers (Supplementary Fig. S2). For a given total optical thickness, a nanolaminate layer with a larger number of sublayers has a higher optical bandgap ${ }^{16}$. Here, four sublayers are used as a compromise between a large bandgap and the overall layer quality. Note that the co-evaporated interfaces have a thickness of $\sim 4 \mathrm{~nm}$. To achieve a high reflectivity at $355 \mathrm{~nm}$ for an angle of incidence of $45^{\circ}$, the reference wavelength $\lambda_{0}$ is $395 \mathrm{~nm}$. The refractive indices of $\mathrm{A}, \mathrm{H}$, and $\mathrm{L}$ at $395 \mathrm{~nm}$ are 1.616, 1.956 , and 1.481, respectively. According to $2 \times\left(n_{\mathrm{A}} d_{0.335 \mathrm{~A}}\right.$ $\left.+n_{\mathrm{H}} d_{0.165 \mathrm{H}}\right)=n_{\mathrm{L}} d_{\mathrm{L}}=n_{\mathrm{A}} d_{\mathrm{A}}=\lambda_{0} / 4$, the physical thicknesses are $d_{4 \mathrm{~L}}=266.80 \mathrm{~nm}, d_{0.335 \mathrm{~A}}=20.47 \mathrm{~nm}, d_{0.165 \mathrm{H}}=$ $8.33 \mathrm{~nm}, d_{\mathrm{L}}=66.70 \mathrm{~nm}, d_{\mathrm{A}}=61.11 \mathrm{~nm}$, and $d_{8.15 \mathrm{~L}}=$ $543.61 \mathrm{~nm}$. The designed $\mathrm{Al}_{2} \mathrm{O}_{3}$ to $\mathrm{HfO}_{2}$ integrated content ratio in the nanolaminate layers is $2.46: 1$. A thick $\mathrm{SiO}_{2}$ overcoat layer $(8.15 \mathrm{~L})$ is used as a protective layer, and the resulting electric (E)-field intensity at the coatingair interface is close to 0 .

For a comparison, the aforementioned NLD coating stack and a TCD coating with a substrate $/ 4 \mathrm{~L}(\mathrm{HL})^{5}(\mathrm{~A}$ $\mathrm{L})^{15} \mathrm{~A} 8.15 \mathrm{~L} /$ air structure are prepared. Obvious sharp diffraction peaks are not observed in the XRD spectra of either the NLD and TCD coatings. Figure 3a, b shows images from high-resolution transmission electron microscopy (TEM) of both coatings, and the lattice is consistent with the selected-area electron diffraction result (see Supplementary Fig. S4). The elemental percentages vs. depth are shown in Fig. 3c. The $\mathrm{Al}_{2} \mathrm{O}_{3}$ to $\mathrm{HfO}_{2}$ integrated content ratio in the nanolaminate layers is $\sim 2.2: 1$, slightly lower than the designed value. $\mathrm{Al}_{2} \mathrm{O}_{3}$ is also observed at the interface between $\mathrm{SiO}_{2}$ and $\mathrm{HfO}_{2}$ in the nanolaminate layers, which is attributed to diffusion and/or preferential sputtering effects. The reflectance spectra of the two coatings are compared in Fig. 3d. The high-reflectivity $(R s \geq 99.5 \%)$ bandwidth of the NLD coating is $38 \mathrm{~nm}$, more than twice that of the TCD coating $(17 \mathrm{~nm})$. In addition, the NLD average transmission is higher in the VIS-NIR region and exhibits smaller ripple amplitudes (Fig. 3e). This result indicates that properly designed NLD structures also have potential as harmonic separators, which require a high reflectivity at one wavelength and a high transmittance at other wavelengths.

The laser damage probabilities as a function of fluence of the two coating structures are compared in Fig. 3f. The damage probability curves show a shape that can, for example, be explained by the presence of two types of defects with different LIDTs. The defect parameters can be extracted using the model developed by Krol et al. ${ }^{27}$. If we assume the existence of two defects with different values of the LIDT $T_{i}$ and area density $D_{i}$ (integrated over the thickness), we obtain the results shown in Table 1. Overall, the NLD coating shows improved damage resistance compared with the damage resistance of the TCD coating stack.

To investigate the damage mechanism, the stress, absorption, E-field distribution, and damage morphology of the coatings are studied. The stresses of the TCD and NLD coatings are determined to be $-160.7 \mathrm{MPa}$ and $-171.1 \mathrm{MPa}$, respectively. The E-field intensity in the NLD coating decays more rapidly with depth than the TCD coating (Fig. 3g). The absorption of the coating stacks is measured using nano-Kelvin calorimetry ${ }^{28}$. At the wavelength of interest $(355 \mathrm{~nm})$, the absorption losses in the NLD coating stack are $\sim 20 \%$ smaller than those in the TCD mirror (Fig. 3h). Consequently, the laser-induced temperature increase in the NLD coating will be lower.

Typical damage morphologies of the NLD and TCD structures are shown in Fig. 4 and suggest the existence of two types of defects. One morphological feature is related to nodules, which produce a pit for an illumination fluence just above the LIDT (Fig. 4i). At larger fluences, the top layers are ablated (Fig. 4j). As the laser fluence further increases, a second type of damage morphology can be observed. This morphology consists of many shallow pits (Fig. 4k), which evolve into larger areas of delaminated films for larger laser fluences (Fig. 4l). The defects that initiate damage features of this second type do not involve nodules. The damage morphology of the second type is observed for fluences of $25.9 \mathrm{~J} / \mathrm{cm}^{2}$ in the TCD coatings (Fig. 4g), while only the first kind of morphology is observed in the NLD coatings for fluences at $32.7 \mathrm{~J} / \mathrm{cm}^{2}$ (Fig. 4b). After laser irradiation with the same fluence above the LIDT, the damaged area in the NLD coating stack is smaller than that in the TCD coating stack.

A finite-element method (FEM) simulation is used to illustrate the effect of nodular defects on the E-field intensity distribution. As an input for the simulation, the nodular defect size is estimated from the FIB cross-sections (Fig. 4). In both the TCD- and NLD-coating stacks, the nodular defects produce an E-field intensification (see Supplementary Fig. S5), especially in the top layers close to air. This result explains why laser damage originates from nodules, and a delamination of top layers occurs as the laser fluence increases. Compared with the TCD coating, the NLD coating exhibits a lower E-field intensification, faster E-field decay with depth and smaller absorption, which are consistent with the observed higher LIDT.

In summary, we have developed and experimentally demonstrated a new class of UVML coatings with an 

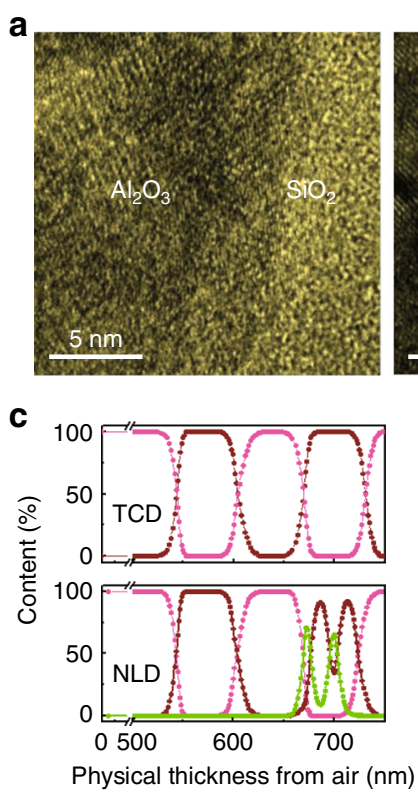

f

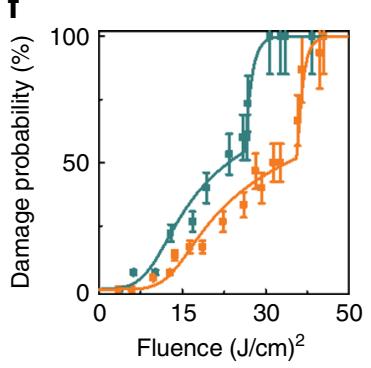

g
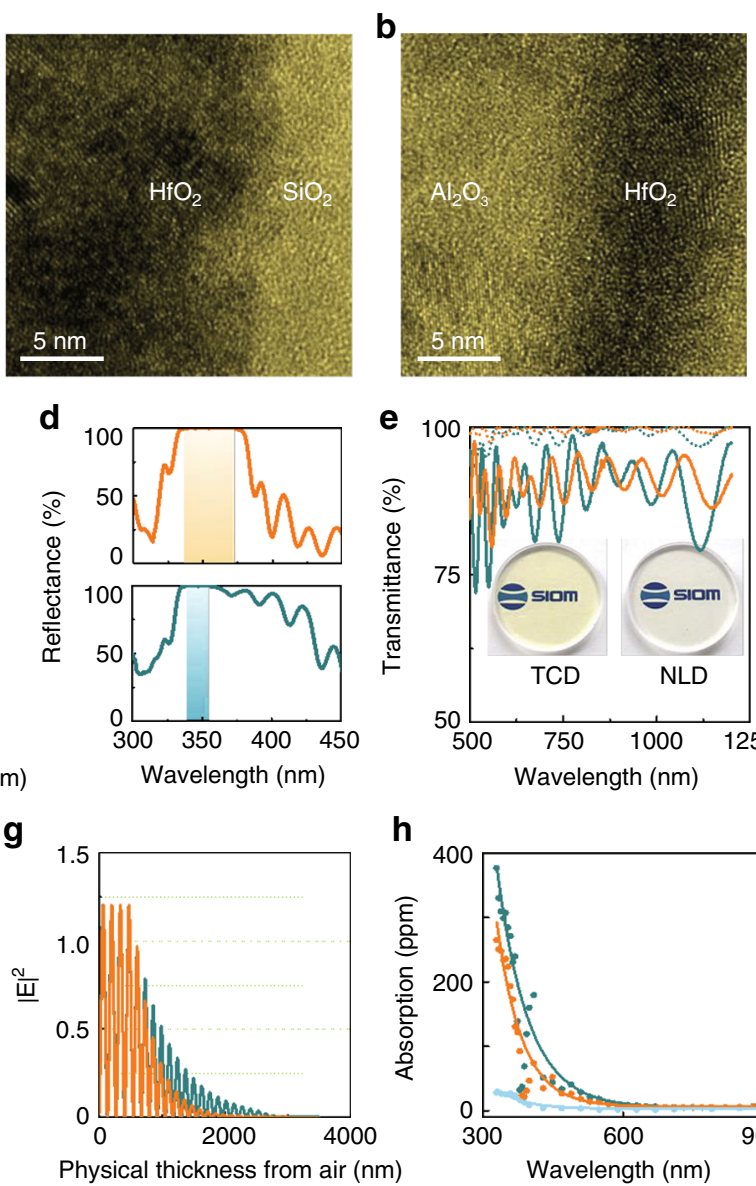

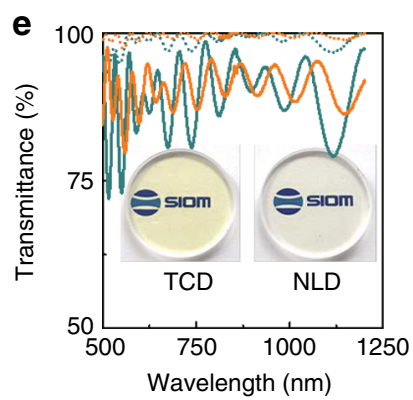

h

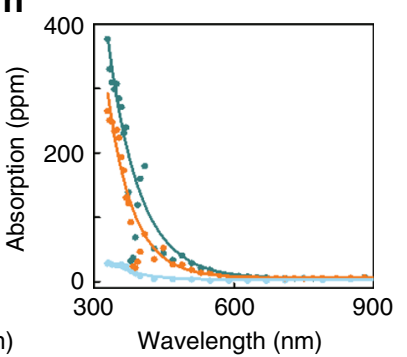

$-\mathrm{Al}_{2} \mathrm{O}_{3}-\mathrm{HfO}_{2}-\mathrm{SiO}_{2}$

— NLD Coating

_ TCD Coating

- Substrate

Fig. 3 The microstructure and optical properties of the TCD and NLD coatings. High-resolution transmission electron microscopy of the (a) TCD and (b) NLD coatings. $\mathbf{c ~} \mathrm{SiO}_{2}, \mathrm{Al}_{2} \mathrm{O}_{3}$, and $\mathrm{HfO}_{2}$ content vs. depth, $\mathbf{d}$ reflectance, and e transmittance spectra (incident angle of $45^{\circ}$, s-polarized light indicated by solid lines, and $p$-polarized light indicated by dotted lines), $\mathbf{f}$ single-pulse damage probability as a function of the input fluence, $\mathbf{g}$ E-field distribution, and $\mathbf{h}$ optical absorption of the TCD and NLD coatings vs. wavelength

Table 1 Extracted defect parameters

\begin{tabular}{lllllll}
\hline $\begin{array}{l}D_{1}(\mathbf{1} / \\
\left.\mathbf{m m}^{2}\right)\end{array}$ & $\begin{array}{l}T_{1}(\mathrm{~J} / \\
\left.\mathbf{c m}^{2}\right)\end{array}$ & $\begin{array}{l}\Delta T_{1}(\mathrm{~J} / \\
\left.\mathbf{c m}^{2}\right)\end{array}$ & $\begin{array}{l}\boldsymbol{D}_{\mathbf{2}}(\mathbf{1 /} \\
\left.\mathbf{m m}^{2}\right)\end{array}$ & $\begin{array}{l}T_{2}(\mathrm{~J} / \\
\left.\mathbf{c m}^{2}\right)\end{array}$ & $\begin{array}{l}\Delta T_{2}(\mathrm{~J} / \\
\left.\mathbf{c m}^{2}\right)\end{array}$ \\
\hline TCD & 6.5 & 10.1 & 6.0 & 200.0 & 25.5 & 1.0 \\
NLD & 6.1 & 13.5 & 6.0 & 298.0 & 35.0 & 1.0 \\
\hline
\end{tabular}

unprecedented combination of properties. The new structure replaces the high- $n$ materials in traditional designs with nanolaminate layers. The proposed method enables UVML coatings with a larger high-reflectivity bandwidth, higher LIDT, and smaller transmission ripples in the VIS-NIR region than traditional designs with a comparable overall thickness. The e-beam-deposited nanolaminate materials can be used for large (meter- scale) UVML coatings. We believe that the described concept opens new avenues for improved UV coatings and can benefit many areas of laser technology that rely on high-quality optical coatings.

\section{Materials and methods Preparation of coatings}

$\mathrm{HfO}_{2}$ and $\mathrm{Al}_{2} \mathrm{O}_{3}$ single-layer coatings, $\mathrm{Al}_{2} \mathrm{O}_{3}-\mathrm{HfO}_{2}$ nanolaminate coatings, and TCD and NLD multilayer coatings are deposited on fused silica substrates using ebeam evaporation. The coating chamber is heated to $473 \mathrm{~K}$ and evacuated to a base pressure of $9 \times 10^{-4} \mathrm{~Pa}$ before deposition. The deposition rates for the $\mathrm{HfO}_{2}$, $\mathrm{Al}_{2} \mathrm{O}_{3}$, and $\mathrm{SiO}_{2}$ layers are $0.1 \mathrm{~nm} / \mathrm{s}, 0.1 \mathrm{~nm} / \mathrm{s}$, and $0.2 \mathrm{~nm} /$ $\mathrm{s}$, respectively. The oxygen pressure of $\mathrm{HfO}_{2}$ and $\mathrm{Al}_{2} \mathrm{O}_{3}$ is $1.3 \times 10^{-2} \mathrm{~Pa}$. Except for the 4L layer $\left(3.0 \times 10^{-3} \mathrm{~Pa}\right)$ in the TCD and NLD coatings, the oxygen pressure of the remaining $\mathrm{SiO}_{2}$ is $5.0 \times 10^{-3} \mathrm{~Pa}$. The co-evaporated 


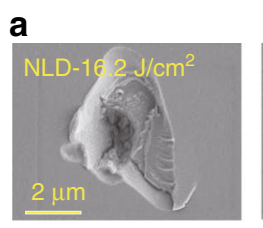

e

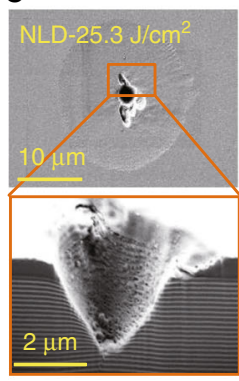

i

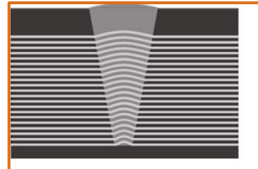

b

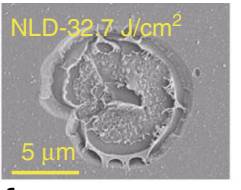

f

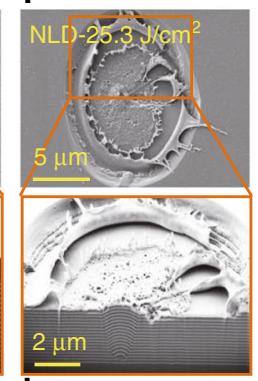

j

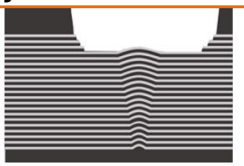

C

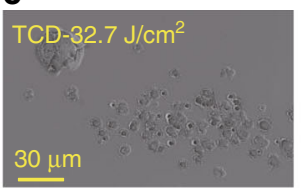

g
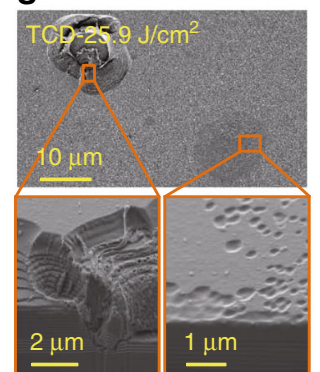

k

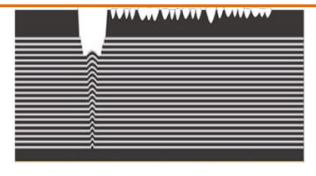

Increasing laser fluence

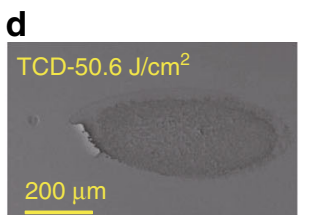

h

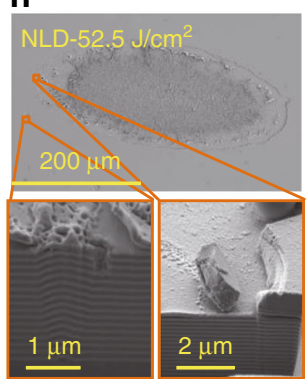

I

Fig. 4 Damage morphologies of the TCD and NLD coatings. a-h Damage sites imaged by SEM and the depth profiles of the marked regions measured by FIB. i-I Schematic diagram of the simulated damage morphologies for increasing laser fluence

interface is obtained by dual e-beam co-evaporation. The details of the co-evaporation system and process are shown in Supplementary Figs. S1 and S2.

\section{Characterization of the coatings}

The transmittance spectra in the range of $150-220 \mathrm{~nm}$ and $220-1200 \mathrm{~nm}$ are measured by a VUV spectrometer (LZH ML 6500) and a UV-visible spectrometer (Perkin Elmer Lambda 1050), respectively. The reflectance spectra in the VIS-NIR region are calculated from the transmission data neglecting absorption. The refractive indices are fitted using commercial thin film software (Essential Macleod). The E-field intensity distributions are obtained from FEM simulations.

The elemental compositions along the depth are determined by X-ray photoelectron spectroscopy (XPS, Thermo Scientific K-Alpha) using a monochromatic Al $\mathrm{K} \alpha(1486.6 \mathrm{eV}) \mathrm{X}$-ray source. The spectra are recorded after every $20 \mathrm{~s}$ of etching with $1 \mathrm{keV} \mathrm{Ar}{ }^{+}$ions.

The surface and cross-section morphologies of the laser-induced damaged sites are obtained by a focused ion beam scanning electron microscope (FIB-SEM, Carl Zeiss AURIGA CrossBeam).

The 1-on-1 LIDT is determined according to ISO 21254 using an $s$-polarized $3 \omega \mathrm{Nd}$ : YAG laser with a Gaussian $\mathrm{TEM}_{00}$, single-longitudinal mode $(355 \mathrm{~nm})$. The pulse shape is Gaussian with a full width at half maximum of
$7.6 \mathrm{~ns}$. The test is performed at an angle of incidence of $45^{\circ}$. The effective beam size on the sample surface is $\sim 0.18 \mathrm{~mm}^{2}$, and 15 sites are tested for each energy fluence. The sample surfaces before (substrate) and 15 days after the deposition are inspected by an interferometer (ZYGO Mark III-GPI) at $632.8 \mathrm{~nm}$ in an environment with a temperature of $23 \pm 1.5^{\circ} \mathrm{C}$ and a relative humidity of $45 \pm 5 \%$. The coating stress is obtained from Stoney's formula. The absorption is measured by an optical nanoKelvin calorimeter ${ }^{28}$ using a tunable light source with a spectral width of $\sim 6 \mathrm{~nm}$, a step size of $5 \mathrm{~nm}$, and an optical illumination power between 30 and $40 \mu \mathrm{W}$.

\section{Acknowledgements}

The authors express their appreciation to Yuan'an Zhao and Yun Cui for the LIDT and FIB measurements, respectively. This work is supported by the National Special Support Program for Young Top-notch Talent; National Natural Science Foundation of China (61975215 and 61505227); Youth Innovation Promotion Association of the Chinese Academy of Sciences; and Shanghai Young Top-notch Talent Program.

\section{Author details}

'Laboratory of Thin Film Optics, Shanghai Institute of Optics and Fine Mechanics, Chinese Academy of Sciences, Shanghai 201800, China. ${ }^{2}$ Center of Materials Science and Optoelectronics Engineering, University of Chinese

Academy of Sciences, Beijing 100049, China. ${ }^{3}$ Hangzhou Institute for Advanced Study, University of Chinese Academy of Sciences, Hangzhou 310024, China. ${ }^{4}$ Key Laboratory of Materials for High Power Laser, Shanghai Institute of Optics and Fine Mechanics, Chinese Academy of Sciences, Shanghai 201800, China. ${ }^{5}$ Department of Physics and Astronomy, University of New Mexico, 
Albuquerque, NM 87131, USA. ${ }^{6}$ CREOL, The College of Optics and Photonics, University of Central Florida, Orlando, FL 32816, USA

\section{Conflict of interest}

The authors declare that they have no conflict of interest.

Supplementary information is available for this paper at https://doi.org/ 10.1038/s41377-020-0257-4.

Received: 1 November 2019 Revised: 13 January 2020 Accepted: 1 February 2020

Published online: 11 February 2020

\section{References}

1. Macilwain, C. Inadequate optics 'threat to US laser facility'. Nature 403, 120 (2000).

2. Extreme light. Nat. Mater. 15, 1 (2016).

3. Fan, Z. W. et al. High beam quality 5 J, $200 \mathrm{~Hz} \mathrm{Nd:YAG} \mathrm{laser} \mathrm{system.} \mathrm{Light} \mathrm{Sci.}$ Appl. 6, e17004 (2017).

4. Norvig, P. et al. 2020 visions. Nature 463, 26-32 (2010).

5. Malobabic, S., Jupé, M. \& Ristau, D. Spatial separation effects in a guiding procedure in a modified ion-beam-sputtering process. Light:: Sci. Appl. 5, e16044 (2016).

6. Jauregui, C., Limpert, J. \& Tünnermann, A. High-power fibre lasers. Nat. Photonics 7, 861-867 (2013).

7. $\mathrm{Hu}, \mathrm{C}$. Q. et al. New design for highly durable infrared-reflective coatings. Light Sci. Appl. 7, 17175 (2018)

8. Xing, $H$. B. et al. Improving laser damage resistance of $355 \mathrm{~nm}$ high-reflective coatings by co-evaporated interfaces. Opt. Lett. 41, 1253-1256 (2016).

9. $\mathrm{Xu}$, L. et al. Improvement of the laser-induced damage threshold of oxide/ fluoride double stack high reflective coatings at $355 \mathrm{~nm}$ by introducing interlayers. Appl. Surf. Sci. 280, 772-775 (2013).

10. Tolenis, T. et al. Next generation highly resistant mirrors featuring all-silica layers. Sci. Rep. 7, 10898 (2017).

11. Hausmann, D. et al. Rapid vapor deposition of highly conformal silica nanolaminates. Science 298, 402-406 (2002).
12. Zhong, L. J. et al. Nanolaminates of zirconia and silica using atomic layer deposition. Chem. Mater. 16, 1098-1103 (2004).

13. Peng, Q. et al. Bi-directional kirkendall effect in coaxial microtube nanolaminate assemblies fabricated by atomic layer deposition. ACS Nano 3, 546-554 (2009).

14. Wickberg, A. et al. Second-harmonic generation from $\mathrm{ZnO} / \mathrm{Al}_{2} \mathrm{O}_{3}$ nanolaminate optical metamaterials grown by atomic-layer deposition. Adv. Optical Mater. 4, 1203-1208 (2016).

15. Willemsen, T. et al. Enhancement of the damage resistance of ultra-fast optics by novel design approaches. Opt. Express 25, 31948-31959 (2017).

16. Willemsen, T. et al. Tunable optical properties of amorphous Tantala layers in a quantizing structure. Opt. Lett. 42, 4502-4505 (2017).

17. Kim, L. $\mathrm{H}$. et al. $\mathrm{Al}_{2} \mathrm{O}_{3} / \mathrm{TiO}_{2}$ nanolaminate thin film encapsulation for organic thin film transistors via plasma-enhanced atomic layer deposition. ACS Appl. Mater. Interfaces 6, 6731-6738 (2014).

18. Heuser, S. et al. 3D 3C-SiC/Graphene hybrid nanolaminate films for highperformance supercapacitors. Small 14, 1801857 (2018).

19. Kahouli, A. et al. Electrical characteristics and conduction mechanisms of amorphous subnanometric $\mathrm{Al}_{2} \mathrm{O}_{3}-\mathrm{TiO}_{2}$ laminate dielectrics deposited by atomic layer deposition. Appl. Phys. Lett. 109, 202901 (2016).

20. Cho, M. H. et al. Dielectric characteristics of $\mathrm{Al}_{2} \mathrm{O}_{3}-\mathrm{HfO}_{2}$ nanolaminates on $\mathrm{Si}$ (100). Appl. Phys. Lett. 81, 1071-1073 (2002).

21. Cheng, X. B. et al. Waterproof coatings for high-power laser cavities. Light Sci. Appl. 8, 12 (2019).

22. $\mathrm{Xu}, \mathrm{N}$. et al. Laser resistance dependence of interface for high-reflective coatings studied by capacitance-voltage and absorption measurement. Opt. Lett. 43, 4538-4541 (2018).

23. Chai, Y. J. et al. Laser-resistance sensitivity to substrate pit size of multilayer coatings. Sci. Rep. 6, 27076 (2016).

24. Cheng, X. B. et al. The effect of an electric field on the thermomechanical damage of nodular defects in dielectric multilayer coatings irradiated by nanosecond laser pulses. Light Sci. Appl. 2, e80 (2013).

25. Grilli, M. L. et al. $\mathrm{Al}_{2} \mathrm{O}_{3} / \mathrm{SiO}_{2}$ and $\mathrm{HfO}_{2} / \mathrm{SiO}_{2}$ dichroic mirrors for UV solid-state lasers. Thin Solid Films 517, 1731-1735 (2009).

26. Tauc, J., Grigorovici, R. \& Vancu, A. Optical properties and electronic structure of amorphous germanium. Phys. Status Solidi (B) 15, 627-637 (1966).

27. Krol, $\mathrm{H}$. et al. Investigation of nanoprecursors threshold distribution in laserdamage testing. Opt. Commun. 256, 184-189 (2005).

28. Roshanzadeh, B., Boyd, S. T. P. \& Rudolph, W. (Submitted for consideration). 